Delgado-Algarra, E. J. y Estepa-Giménez, J. (2016). Ciudadanía y memoria histórica en la enseñanza de la historia: análisis de la metodología didáctica en un estudio de caso en ESO. Revista de Investigación Educativa, 34(2), 521-534. DOI: http://dx.doi.org/10.6018/rie.34.2.224891

\title{
Ciudadanía y memoria histórica en la enseñanza de la historia: análisis de la metodología didáctica en un estudio de caso en ESO
}

\section{Citizenship and memory in history teaching: analisys of the didactic methodology in a case study in ESO}

\author{
Emilio José Delgado-Algarra* y Jesús Estepa-Giménez** \\ *Departamento de Comunicación y Educación. Facultad de Humanidades y Ciencias Sociales. \\ Universidad Loyola Andalucía (España). \\ **Departamento de Didáctica de las Ciencias y Filosofía. Facultad de Educación. Universidad de Huelva (España)
}

\begin{abstract}
Resumen
Mediante un estudio de caso, analizamos cómo se enseña ciudadanía y las dimensiones de la memoria en una clase de Geografía e Historia de $4^{\circ}$ de ESO. Para ello, con el apoyo del programa de análisis cualitativo "Atlas ti", se lleva a cabo una categorización de las informaciones transcritas de la entrevista de concepciones al docente, del registro de audio sobre el desarrollo de las clases, del material curricular utilizado como guía principal en el aula, de los cuestionarios de percepción discente y de la entrevista final de reflexión docente. Con estas cinco fuentes de información se realiza una doble triangulación para contrastar las interpretaciones del docente, el investigador y los estudiantes. El caso de estudio potencia el debate en el aula y da una importancia destacada a la memoria histórica, estableciendo un vínculo intenso entre el recuerdo de terceras personas y la enseñanza de la Historia.

Palabras clave: enseñanza de la historia; educación para la ciudadanía; memoria histórica; metodología didáctica.

Correspondencia: Emilio José Delgado Algarra, Departamento de Comunicación y Educación. Universidad Loyola Andalucía (Sevilla). E-mail: emiliojose.delgadoalgarra@gmail.com. Jesús Estepa Giménez, Departamento de Didáctica de las Ciencias y Filosofía. Universidad de Huelva. E-mail: jestepa@uhu.es
\end{abstract}




\begin{abstract}
Using the Study Case method, we analyze how both, the concept of citizenship and the different dimensions of memory, are taught in the fourth course of Secondary Education, concretely within the subject of Geography and History. With the support of the qualitative analysis program Atlas.ti, we carry out a categorization of the information transcribed from the interview given to the teacher on his previous concepts on this issue. Furthermore, we analyze the audio transcription of the recorded classes, the curricular material used as principal guide in class, the questionnaires given to the students on their perception of the classes, and the final interview with the teacher feedback on the same classes. Taking as basis these five sources of information, we carry out a double triangulation in order to contrast the interpretations of the teacher, the researcher, and the students. The Study Case enhances classroom discussion and gives a prominent role to historical memory, establishing an strong bond between the rememberances of the people involved in certain historical situations and the teaching of History.

Keywords: teaching of history; citizenship education; historical memory; didactical methodology.
\end{abstract}

\title{
Introducción
}

En este artículo se va a abordar la educación para la ciudadanía a través de la memoria histórica a partir del análisis de las clases de un profesor de secundaria, lo que constituye una parte de un estudio más amplio realizado en la Tesis Doctoral "Educación para la ciudadanía en la enseñanza de las Ciencias Sociales y su vinculación con las dimensiones de la memoria. Estudio de caso en ESO" (Delgado Algarra, 2014), centrándonos en los bloques del sistema de categorías vinculados con cómo se enseña educación para la ciudadanía y memoria en la enseñanza de las Ciencias Sociales. A continuación, tras hacer una breve presentación del marco teórico y del diseño de investigación centramos nuestro análisis de la praxis en la labor realizada por el docente en el aula con su alumnado de $4^{\mathrm{o}}$ de ESO durante el desarrollo de las unidades didácticas 9 (España: primer tercio del siglo XX) y 14 (España: Dictadura y Democracia) del libro de texto de Ciencias Sociales del mencionado curso de la editorial Oxford Education; estas unidades, respondiendo a una organización cronológica de la materia, se fusionaron y desarrollaron conjuntamente entre el 8 de abril y el 21 de mayo de 2013 a lo largo de 12 sesiones.

\section{Marco teórico}

Se han realizado numerosas investigaciones en torno a la enseñanza de la ciudadanía en el ámbito internacional (Arthur, Davies \& Hahn, 2008) y, en menor medida, como contenido de las Ciencias Sociales, Geografía e Historia (De Alba, García Pérez y Santisteban, 2012; Estepa, 2009). Sin embargo, son más escasas las que relacionan la enseñanza de la Historia y la memoria, destacando, entre otros, los estudios de Carretero, Rosa y González (2006) para España y América, de Ienaga (2001) para Japón, de Lässig (2009) respecto a Alemania y de Mattozzi (2008) en referencia a Italia. En cuanto a la educación ciudadana, desde nuestra perspectiva crítica basada en una 
escuela entendida como motor de cambio social, pensamos que resulta destacable la asunción de un modelo de democracia radical donde no existen elementos considerados inamovibles y todo puede ser trabajado y construido mediante debate. En otras palabras, este modelo de ciudadanía -representado entre otros por la politóloga Mouffe (1999) - entiende que ésta se construye sobre la base de los posicionamientos ideológicos de los sujetos y la lucha entre dichos posicionamientos, de manera que la reivindicación y el conflicto son considerados como aspectos positivos en el ámbito político y social. De esta manera, tanto la ciudadanía como la memoria deben entenderse en el seno del diálogo y del intercambio de ideas, en el cual las personas deben posicionarse firmemente y estar abiertas a modificar dichos posicionamientos frente a una buena argumentación. Así, el modelo de escuela crítica y democrática aspira a construir un modelo de ciudadano capaz de tomar decisiones en el seno de una democracia participativa (Schugurensky, 2009; Schugurensky \& Myers, 2003); sin embargo, cabe matizar que suelen existir contradicciones entre ideales democráticos y aulas no democráticas, entre declaraciones pedagógicas del profesorado y la práctica docente real.

La memoria, por su parte, debe ser entendida como un recuerdo cargado de valores subjetivos y relacionado con los momentos conflictivos de nuestra historia reciente, definiéndose a raíz de la experiencia personal de cada individuo en el seno de un grupo social determinado. En torno a ella, la institución educativa se enfrenta al problema de la adopción de una doble postura (Rinaldi, 2008): la escuela es la institución en la que más debería transmitirse y procesarse la memoria; sin embargo, es también la institución que más se debe esforzar por diluir los efectos negativos y dudosos derivados de los excesos de la memoria. La memoria histórica, que tanta conexión guarda con la formación cívica, debe tratarse desde todos los puntos de vista posibles (Ienaga, 2001; Mejía, 2009). Hay que recordar que las concepciones de ciudadanía, historia y sociedad están determinadas, en gran medida, por el factor humano; una variable extraña más que significativa que obliga a contemplar, por ejemplo, la visión de un conflicto desde el punto de vista de las diferentes partes implicadas. Sin embargo, cuando alguien habla de memoria histórica, suele entenderse que existe un posicionamiento ideológico definido. Así en Japón, por ejemplo, al igual que en España, existe una tendencia al análisis crítico de la memoria histórica por parte de los sectores progresistas, mientras que, de manera divergente, se observa una tendencia hacia la ocultación y a la revisión justificadora de la memoria histórica por parte de los sectores conservadores (Nozaki, 2001, 2008), persiguiendo con ello el objetivo de desmentir las agresiones y eludir responsabilidades ante las "hazañas patrióticas" del ejército imperial japonés en los conflictos del pasado reciente.

Para la realización de la presente investigación se ha tomado como referente el modelo multidimensional de la memoria de Mainer (2010) y Cuesta (2011): memoria individual, memoria social, memoria histórica, memoria conflictiva y memoria selectiva. La decisión de tomar este modelo multidimensional como referencia en vinculación con la educación para la ciudadanía se debe, en parte, a que la mayoría de las investigaciones sobre memoria en la enseñanza de la Historia suelen centrarse en su dimensión histórica y conflictiva y a que, en raras ocasiones, se atiende a la vinculación entre memoria y educación ciudadana. 


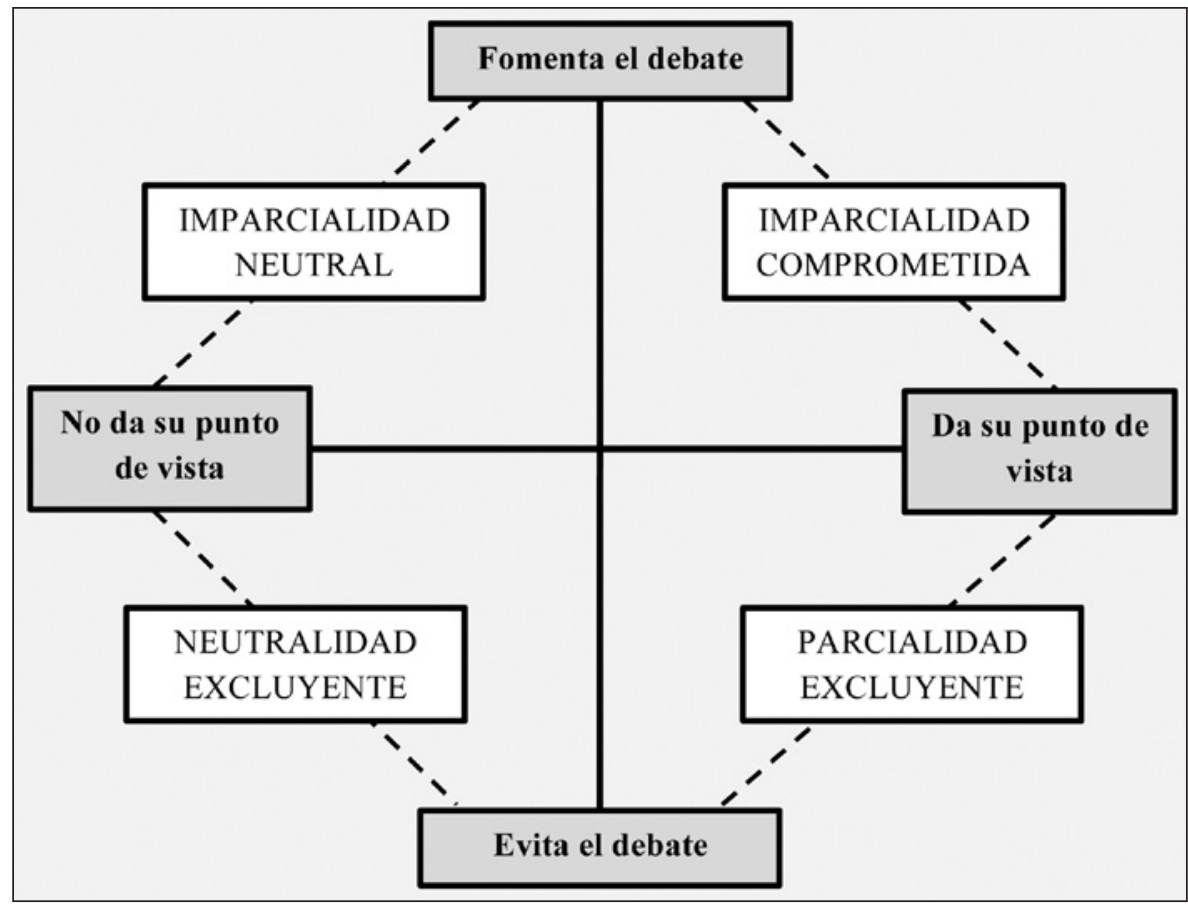

Figura 1. Actitud de los profesores frente a la inclusión de problemas sociales relevantes en el aula (Delgado Algarra, 2014).

Respecto a la metodología didáctica, que es la categoría de análisis en el presente estudio de caso, partimos de una hipótesis de progresión elaborada según los presupuestos del Proyecto IRES (Porlán y Rivero, 1998), de modo que los diferentes itinerarios que puede seguir un docente en su desarrollo profesional tienen en cuenta tanto el conocimiento profesional del profesorado como sus concepciones. En este modelo, la evolución del docente pasa de no contemplar la metodología didáctica como un problema profesional, a un nivel de desarrollo, que consideramos deseable, en el cual se implementan estrategias metodológicas centradas en la investigación escolar de problemas socioambientales, donde la participación es negociada de manera que el alumnado, progresivamente, va tomando más decisiones; todo ello pasando por niveles intermedios caracterizados por el diseño de secuencias cerradas y respuestas prescritas por el profesor, por un lado, o de desarrollo de actividades improvisadas y basadas en el descubrimiento espontáneo de la realidad, por otro. Además, aunque la actitud de los profesores frente a los problemas socialmente conflictivos ha sido clasificada en los cuatro tipos que se representan en la figura 1 (Kelly, 1986; López Facal, 2011), consideramos imprescindible una adecuada formación docente y la asunción por parte de éste del modelo de imparcialidad comprometida, ya que dicho modelo implica el fomento de los debates en el aula y que el docente dé su opinión en los mismos. 


\section{Método}

\section{Problemática}

El problema central de la investigación es “¿Qué y cómo se enseña educación para la ciudadanía en Ciencias Sociales, Geografía e Historia en Educación Secundaria Obligatoria, y qué relación guardan los contenidos y la metodología didáctica con las dimensiones de la memoria?". Aunque en este artículo vamos a centrarnos en las problemáticas específicas 2 y 3 , a continuación se presentan las cuatro que permitieron dar respuesta al problema central:

- Problema específico 1: “¿Cuál es la concepción de ciudadanía que tiene el profesor y qué reflejo tiene en su práctica?".

- Problema específico 2: “¿Qué se enseña de educación para la ciudadanía en Ciencias Sociales, Geografía e Historia?".

- Problema específico 3: “¿Cómo se enseña educación para la ciudadanía en Ciencias Sociales, Geografía e Historia?".

- Problema específico 4: “¿Qué y cómo se trabajan las diferentes dimensiones de la memoria en las clases de Ciencias Sociales, Geografía e Historia?".

\section{Contextualización del caso}

El caso de estudio se encuentra representado por el docente JMT, profesor de Geografía, Historia y Educación para la Ciudadanía de los cursos 1ํㅡ - 4⿳⺈ de ESO en el centro IES “La Orden" de la ciudad de Huelva (Andalucía - España). La oferta educativa de este centro va desde $1^{\circ}$ de ESO hasta $2^{\circ}$ de Bachillerato, incluyendo estudios de formación profesional; sin olvidar que en él trabajan 66 personas, 55 profesores y 11 profesionales de administración y servicios.

En cuanto al contexto geográfico y socioeconómico del IES La Orden, el centro educativo se encuentra próximo al parque Moret y está rodeado por los barrios de Santa Marta y La Orden. En el barrio de La Orden, con más de 20.000 habitantes, encontramos zonas de alta conflictividad social, donde el $60 \%$ de los adultos carece de estudios y más de 1/3 (dentro de dicho porcentaje), según la oficina del Defensor del Onubense, se declara analfabeto.

\section{Sistema de categorías}

Tanto para orientar el proceso de recogida y organización de información como para su análisis, se elaboró un sistema de categorías específico para nuestra investigación que se inspira en el que se diseñó para el Proyecto I+D+i "el Patrimonio y su enseñanza: análisis de recursos y materiales para una propuesta integrada de Educación Patrimonial" (Estepa, 2013) y cuya estructura específica bebe de múltiples fuentes, entre las que se encuentra la International Association for the Evaluation of Educational Achievement (Schulz, Fraillon, Ainley, Bruno \& Kerr, 2008), para lo referente a educación ciudadana; y Fedicaria y el Proyecto Nebraska (Cuesta, 2011; Mainer, 2010), para todo lo relacionado 
con las dimensiones de la memoria. Las categorías del sistema son cuatro: tipos de ciudadanía (CIU), qué se enseña de educación para la ciudadanía en Ciencias Sociales (QEC), cómo se enseña educación para la ciudadanía en Ciencias Sociales (CEC), qué y cómo se enseñan las dimensiones de la memoria en la educación ciudadana (DIM). Sin embargo, en este artículo nos centraremos en las categorías CEC y en los aspectos vinculados con la metodología de DIM. Atendiendo a las subcategorías e indicadores, y sin entrar en los descriptores por cuestiones de espacio, el sistema se resume como se muestra en la Tabla 1.

Tabla 1

Categorías y subcategorías vinculados a cómo se enseña ciudadanía y memoria en el área de Ciencias Sociales, Geografía e Historia

\begin{tabular}{ll}
\hline Categorías & Subcategorías \\
\hline $\begin{array}{l}\text { Cómo se enseña educación para la ciudadanía } \\
\text { en CCSS (CEC) }\end{array}$ & $\begin{array}{l}\text { Compromiso del profesor (COP) } \\
\text { Materiales curriculares (MAT) }\end{array}$ \\
& Dimensión individual (DIN) \\
$\begin{array}{l}\text { Qué y cómo se enseñan las dimensiones de } \\
\text { la memoria en la educación ciudadana (DIM) }\end{array}$ & $\begin{array}{l}\text { Dimensión histórica (DHI) } \\
\text { Dimensión conflictiva (DCO) }\end{array}$ \\
& Dimensión selectiva (DSE) \\
\hline
\end{tabular}

\section{Instrumentos de recogida de información}

\section{Entrevista inicial de concepciones y entrevista final de reflexión docente}

El número de entrevistas al docente fue de dos, una de concepciones (y selección del caso), al inicio del proceso de recogida de información; y otra de reflexión sobre su práctica (donde el docente responde a las percepciones del alumnado sobre sus clases en el cuestionario discente), al final del proceso de recogida de información. La duración de las sesiones de entrevista fue de unos 45 minutos cada una en horario complementario. Atendiendo a la tipología de Patton (1987), la primera entrevista es de carácter abierto y no estructurado y se diseña tomando como referente el sistema de categorías. La segunda entrevista, sin embargo, requiere tomar aspectos de la práctica real del docente, por lo que se diseñan unas cuestiones orientativas en la etapa final del proceso de recogida de información, manteniendo la coherencia con el sistema de categorías antes mencionado. 


\section{Análisis de materiales curriculares}

El modelo de registro y organización de la información visto anteriormente es similar al que se usó en el análisis de los materiales curriculares donde, además del libro de texto de Ciencias Sociales de 4o de ESO de Oxford Education (Díaz Rubiano, Fernández Armijo, Jiménez Maqueda, Del Pino y Vidal, 2008), se usaron los artículos "La derrota más amarga del Ejército español" (El País, 19/07/2011) y "Gaspar cavó su propia fosa" (El País, 30/03/2013), la remasterización del noticiario de la FOX del año 31 "Memoria recuperada de la República"; además de otros textos para el análisis crítico como cartillas de racionamiento, documento de propaganda de la autarquía, etc.; todo ello sin olvidar fuentes orales, como el discurso de Alfonso XIII arengando a las tropas en la Guerra de Marruecos y otro archivo de audio con diferentes himnos.

\section{Observación de la praxis docente}

Como indica Evans (2010), "la forma de registrar y luego reducir y/o codificar los resultados es importante para que cumpla su propósito" (p. 65). En este sentido, las clases fueron grabadas mediante grabadora de audio, igualmente, se tomaron notas puntuales en el diario del investigador; ya que éste estaba presente en la última mesa como miembro no participante. La información registrada mediante grabación y transcrita, al igual que la del resto de instrumentos de la fase cualitativa, se categoriza mediante el programa Atlas.ti.

\section{Cuestionario sobre percepción discente}

Pese a que la presente investigación se centra fundamentalmente en el docente, conocer el pensamiento del alumnado en relación conel desarrollo de las clases nos permite contrastar la interpretación emergente de la primera triangulación (entrevista inicial - observación de aula - materiales curriculares). Para ello, se aplica un cuestionario sobre pensamiento discente a los 14 alumnos del aula observada, en cuyo diseño se toma como fuente de referencia el desarrollo de las clases.

\section{Temporalización de la recogida de información}

Una vez preseleccionado el caso con la ayuda del cuestionario inicial de concepciones docentes $\left(\mathrm{C}_{0}\right)$ dentro de la fase cuantitativa, se pasa a la fase cualitativa. Dentro de dicha fase, en un periodo inicial, se realiza la entrevista sobre concepciones (E1) que nos permite seleccionar definitivamente el caso de estudio, posteriormente, se inicia la observación de la praxis docente (O1) y la revisión de los materiales curriculares (M1); durante el proceso de recogida de información, se sigue obteniendo información de O1 y M1; y en el periodo final, además de cerrar O1 y M1, se aplica un cuestionario de pensamiento discente a los alumnos sobre su percepción de los contenidos trabajados y sobre el desarrollo de las clases (C1) y se lleva a cabo una entrevista de autoreflexión del profesor sobre su praxis (E2), donde interpreta el porqué de las respuestas de sus alumnos. 
Tabla 2

Temporalización del proceso de recogida de información en la investigación.

\begin{tabular}{|c|c|c|c|c|c|c|}
\hline \multicolumn{2}{|c|}{ MOMENTOS } & \multicolumn{4}{|c|}{ INSTRUMENTOS } & SUJETOS \\
\hline \multicolumn{2}{|c|}{ Previo a la selección del caso } & \multicolumn{4}{|c|}{$\mathrm{C}_{0}$} & Docentes \\
\hline \multirow{3}{*}{$\begin{array}{l}\text { Posterior a la } \\
\text { selección del } \\
\text { caso }\end{array}$} & Recogida inicial & & & \multirow{3}{*}{$\mathrm{O} 1$} & \multirow{3}{*}{ M1 } & Docente \\
\hline & Recogida continua & & & & & $\begin{array}{l}\text { Docente } \\
\text { Materiales }\end{array}$ \\
\hline & Recogida final & E2 & $\mathrm{C} 1$ & & & $\begin{array}{l}\text { Docente } \\
\text { Discente }\end{array}$ \\
\hline
\end{tabular}

TRIANGULACIÓN

\section{Transcripción de la información}

A la hora de organizar las informaciones extraídas de las transcripciones, y para comprender la categorización de las evidencias incluidas en el apartado de "resultados y discusión de datos", en todos los casos, se comienza con el acrónimo del instrumento (ver sub-apartado de temporalización). En el caso de las entrevistas continúa con el número de pregunta y en el del cuestionario el numero asignado al estudiante y el de la cuestión; en cuanto al libro de texto, va seguido del número de página; $\mathrm{y}$, en relación con la observación de aula, continúa con el número de sesión y una letra que representa el intervalo temporal en base a la tabla 3:

Tabla 3

Representación de los intervalos temporales de la obervación de aula.

\begin{tabular}{cccc} 
& & \multicolumn{2}{c}{ Intervalo de tiempo aproximado (minutos) } \\
\cline { 3 - 4 } & A & Desde & Hasta \\
\hline \multirow{4}{*}{ Código } & B min. & $10 \mathrm{~min}$. \\
& B & $10 \mathrm{~min}$. & $20 \mathrm{~min}$. \\
& C & $20 \mathrm{~min}$. & $30 \mathrm{~min}$. \\
& D & $30 \mathrm{~min}$. & $40 \mathrm{~min}$. \\
& E & $40 \mathrm{~min}$. & Final \\
\hline
\end{tabular}

\section{Análisis de la información: la doble triangulación}

La investigación que se presenta tiene un enfoque multimetódico (Glaser y Strauss, 1967), lo que nos permite contrastar la intersubjetividad y asegurar la credibilidad de nuestro proceso de investigación cualitativa. Además, como recomienda Patton (2002), nuestra triangulación implica el uso de fuentes de información múltiples, lo cual nos permite superar las debilidades teóricas y analíticas que podrían darse ante la recogida de información individual. El modelo de triangulación multimetódica y de múltiples fuentes, que se representa en la figura 1, lo hemos denominado doble 
triangulación. Mientras en la primera triangulación (E1 - O1 - M1) se lleva a cabo un proceso de interpretación para destacar las convergencias y divergencia existentes entre las concepciones del docente y su práctica, bajo el paraguas del sistema de categorías; en la segunda triangulación (C1 - O1 - E2), reforzamos la validez y la fiabilidad de nuestras interpretaciones con apoyo de la percepción que los estudiantes tienen del desarrollo de las clases y la interpretación que el docente hace de dichas percepciones (figura 2).

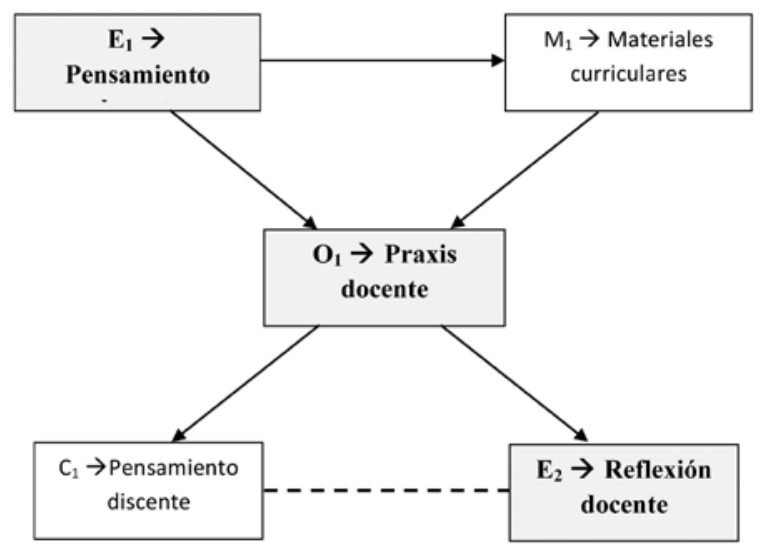

Figura 2. Esquema de la doble triangulación utilizada en el estudio.

\section{Resultados y discusión de los datos}

Estos resultados se extraen a partir de la respuesta al problema específico 3 " ¿Cómo se enseña educación para la ciudadanía en Ciencias sociales, Geografía e Historia?” y a las cuestiones vinculadas con la metodología didáctica en el problema específico 4 "¿qué y cómo se trabajan las diferentes dimensiones de la memoria en las clases de Ciencias Sociales, Geografía e Historia?".

Respecto a la subcategoría compromiso del profesor (COP), siguiendo el planteamiento de De Miguel (2015), nos encontramos ante un docente comprometido con las temáticas puestas a debate, nunca dice si una opinión está bien o mal, sencillamente busca que el alumno sepa defender su posicionamiento en base a preguntas que orientan las intervenciones y permiten a los alumnos afinar en argumentaciones. Existen muchos momentos expositivos, pero el docente hace preguntas constantemente para que el alumnado participe e igualmente da su opinión cuando lo estima necesario, posicionándose en un modelo de imparcialidad comprometida. De este modo, cuando están hablando sobre las características de la República, el docente indica que "por lo menos yo pienso así, que en los países desarrollados pensamos que la república es algo como muy democrático" (O.1.B). También, cuando plantea cuestiones sobre el tema de la raza en los discursos de Alfonso XIII, el docente dice: "Franco, posteriormente, eso lo utilizará mucho también, lo de la raza, como si nosotros fuéramos diferentes al resto de la especie humana, o sea, en vez de unir estamos como separando (...) por lo menos yo lo veo 
así, no sé si vosotros lo veis igual" (O.1.C); de esta forma, abre la posibilidad de réplica por parte del alumnado sin que ello implique ocultar su propia opinión.

Así mismo, cuando los alumnos le preguntan por el partido político EQUO, el profesor indica: "si no recuerdo mal creo que tiene un solo escaño, pero bueno, fue la primera vez que se presentaban, es cuestión de tiempo ¿no?. Creo..." (O.11.C); en cualquier caso, pide a los alumnos que decidan libremente a quién votarían si pudiesen votar y hace referencia a 3 alumnos que no han votado, que finalmente deciden votar en blanco, algo a lo que casi al final de la última sesión se acogen 3 alumnos más (O.12.D). Finalmente, durante la simulación de la intención de voto, el docente indica a los alumnos que dicen no tener ideología que "aquí se supone que todo el mundo tiene ideología, tú dices que no tienes ideología" (nota: mira a una alumna que decía no tener ideología), y añade “ ¿hay algo que no te gusta de lo que está haciendo el Gobierno hoy en día?” (O.12.C).

Los datos del cuestionario del alumnado también nos permiten adscribir al docente al modelo de imparcialidad comprometida, ya sus 14 alumnos, en la cuestión 13 "Marca con una " $X$ " qué idea se corresponde más con el desarrollo de las clases de Historia en $4^{\circ}$ de ESO", señalan la opción "Mi profesor nos permite debatir y da su opinión". En la cuestión 14 "Durante el desarrollo de las clases, ¿te sientes libre para opinar? ¿Por qué?", el 100\% del alumnado afirma sentirse libre para opinar, destacando preferentemente el interés que tiene el docente por sus opiniones y la libertad de expresión. Finalmente, en relación con la cuestión 15 “Piensas que tu profesor quiere una única opinión o plantea la posibilidad de que puedan existir diferentes opiniones en los debates?", ningún estudiante siente que el docente quiera una única opinión. En este sentido, en el pensamiento, praxis y reflexión docente se refleja la importancia de los debates en una asignatura como Historia, aspecto justificable por investigaciones como la realizada por Pagés y Oller (2007) con alumnado de $4^{\mathrm{o}}$ de ESO, donde se destacan las potencialidades de los métodos interactivos, la inclusión de problemas relevantes y la necesidad de conectar continuamente pasado - presente - futuro de cara a minimizar los estereotipos y prejuicios.

En coherencia con la investigación de Santisteban (2007) sobre cómo se aprende el tiempo histórico, hemos comprobado que el docente defiende abiertamente e implementa en la práctica, una visión de la enseñanza de la Historia donde se conecte pasado, presente y, en ocasiones, futuro. Esto se ha visto reflejado en el alumnado tanto en el desarrollo de las clases como en los cuestionarios de pensamiento discente. Respecto a esto último, en la cuestión 16 "¿Tu profesor compara el presente con el pasado? Si la respuesta es "sí" ¿Qué beneficios piensas que esa comparación aporta a tu formación como ciudadano?", únicamente 2 estudiantes indican que el profesor no vincula pasado, presente y futuro; sin embargo, las explicaciones de los estudiantes que responden afirmativamente no son significativas.

En cuanto a los materiales curriculares (MAT), el libro de texto es entendido a modo de guía principal de la acción en el aula, sin embargo, éste se ha visto complementado por el audio del discurso de Alfonso XIII para profundizar en las preocupaciones políticas y militares de España bajo su reinado, la información del artículo "la derrota más amarga del Ejército español" en relación con la Guerra de Marruecos, la del vídeo "Memoria recuperada de la República" para la proclamación de la misma, textos sobre la autarquía y cartillas de racionamiento, para profundizar en los ideales y en 
los problemas socioeconómicos de la España de la posguerra, y el artículo "Gaspar cavó su propia fosa" en relación con la represión franquista.

Uno de los aspectos observados en relación con esta subcategoría, es que durante el desarrollo de las clases el docente trata de conectar lo que se ve en el libro de texto con los intereses del alumnado. Algunos de los intentos del docente de servir de puente entre el libro de texto y los intereses del alumnado los encontramos en los siguientes ejemplos: en cuanto a los resultados electorales de 1931 (pág. 178), el docente busca relacionar los partidos que ahí aparecen con los partidos de la actualidad conocidos por el alumnado (O.4.C); al referirse al Plan Marshall, que también aparece en el libro de texto (pág. 169), pregunta a su alumnado si han visto la película "Bienvenido Mr. Marshall" (O.8.E), aunque tal película resulta completamente desconocida para el alumnado; finalmente, cuando el docente habla del intento de golpe de estado del 23 de febrero de 1981 (pág. 276) y la famosa frase de Tejero ("quieto todo el mundo") (O.11.B), pregunta a su alumnado si alguien había visto la película que emitieron hace poco sobre el suceso. En cualquier caso, debemos entender que, de cara a este descriptor, el intento de conectar con los intereses del alumno por parte del docente es independiente del carácter convencional y enciclopedista del libro de texto, que se usa como guía principal durante el desarrollo de las clases.

Las dimensiones de la memoria (DIM), por su parte, adquieren un peso importante durante las sesiones presenciales $\mathrm{y}$, aunque muchas veces se habla de hechos históricos objetivos y secuenciados cronológicamente (especialmente al inicio de las clases, a modo de resumen de la sesión anterior), la introducción de las opiniones del alumnado y del docente tienen una gran presencia a lo largo de las sesiones, al igual que las reflexiones en torno a las causas de los sucesos acontecidos. Dentro de la dimensión individual, es destacable la importancia que se da a los recuerdos del alumno y a los recuerdos de terceras personas a través de fuentes orales y textuales. Dentro de la dimensión social, destaca la vinculación de los recuerdos con el género (hombre - mujer), las prácticas socio-históricas (nacionales - republicanos), etc.; dentro de la dimensión histórica, se tratan los sucesos históricos de manera cronológica, acompañados de las opiniones respecto a dichos sucesos; dentro de la dimensión conflictiva, el docente incluye los problemas reales e históricos, además de sus interconexiones y analogías; $\mathrm{y}$, finalmente, dentro de la dimensión selectiva, destaca el recuerdo a las víctimas de la Guerra Civil española y del franquismo, estando éste muy presente en gran parte de las sesiones.

Se registran evidencias que vinculan la educación ciudadana con la inclusión de la memoria en la enseñanza de la historia, estos resultados convergen con los emergentes del informe de McCary (2002) "Investigating the Use of Narrative in Affective Learning on issues of Social Justice", donde se concluye con que las enseñanzas conectadas con la memoria personal fomentan la empatía y la caída de estereotipos, lo que desemboca en el desarrollo de conductas coherentes con la justicia social y con respecto a aquellos que han sido silenciados u olvidados. Prueba de esta influencia la hemos observado al analizar la pregunta 11 de los cuestionarios, respecto a la pertinencia del recuerdo o el olvido de las víctimas de la dictadura franquista, 12 de los 14 estudiantes reconocen críticamente las ventajas del recuerdo porque "nos hacen reflexionar para no cometer los mismos errores en el futuro" (C.10.11), aspecto que, con otras palabras, es compartido por la mayoría de los estudiantes. Dos alumnos, sin embargo, se decantaron por el 
olvido debido a que "los ineptos sólo sacan rencores y odios de ellas y porque el hecho de analizarlas no sirve para nada bueno (...)" (C.1.11 alumno 1), matizando además que ambos estudiantes justifican el olvido debido a la imposibilidad de cambiar el pasado. Destacamos la respuesta del alumno 1, quien muestra una visión muy sesgada y parcial de lo que supone recordar cuestiones vinculadas con la memoria. De hecho, al referirse a los que desean conocer la memoria como los "ineptos", que "sólo sacan odios y rencores", indica la existencia de un miedo o un sentimiento de rechazo total a que la gente tenga libertad para acceder a conocer lo que ocurrió en realidad, algo que se pone en evidencia cuando añade que el análisis de la memoria de las víctimas de la Guerra Civil y la Dictadura franquista "no sirve para nada bueno". Este posicionamiento es coherente con el posicionamiento franquista de invisibilización de media España a la que se hace alusión en el desarrollo de las clases. Otra alumna no es capaz de posicionarse porque, según ella, "olvidar hace que la gente deje de sufrir y recordar para saber qué paso y para que seamos conscientes de la suerte que tenemos" (alumna 3).

En cuanto al recuerdo, casi todas las aportaciones se justifican en no repetir en el futuro errores del pasado. El recuerdo de la memoria de las víctimas de los periodos precedentes en este descriptor se refleja a través de la inclusión de la memoria de la familia del docente en el aula. Esto es, las experiencias de su madre, su padre, su tío y su abuelo sirven de ejemplo muy gráfico, sin olvidar recuerdos de otras personas como el de Gaspar y su familia a través del análisis de artículos periodísticos, o la memoria de personas ilustres como Blas Infante o Federico García Lorca, a través del análisis de los textos que encontramos en el libro de texto.

Finalmente, cabe matizar que, pese a que se puso en marcha una actividad para que los estudiantes pudiesen hablar de experiencias de sus familiares en relación con la Guerra Civil y/o la dictadura franquista, las intervenciones discentes fueron escasas. En relación con esto, en la entrevista final, el docente hace las siguientes interpretaciones de la situación "sus padres, seguramente, ya no vivieron la Dictadura, porque nacieron posteriormente, eran muy pequeños cuando la dictadura todavía existía" $\mathrm{y}$, añade como segunda posibilidad, "En España también hubo como una especie de amnesia social de gran parte de la población, sobre todo a partir de la Dictadura. De una época como la de la II República y la Guerra Civil que había que superar y que había que olvidar y ello hace, supongo, que sus padres o incluso sus abuelos, quizás, no quisieran hablar de ese periodo".

\section{Conclusiones}

Dentro del cómo se enseña, como hemos podido apreciar, nos encontramos ante un docente comprometido con las temáticas puestas a debate, que busca que el alumnado sepa defender su posicionamiento ante las preguntas que orientan las intervenciones. En este sentido, en el pensamiento, praxis y reflexión docente se refleja la importancia de los debates y la memoria histórica, estableciendo un vínculo intenso entre el recuerdo de terceras personas y la enseñanza de la Historia

De los cuatro niveles que recogíamos en nuestro modelo de desarrollo profesional respecto a la metodología didáctica, debemos situar la acción docente a caballo entre los dos niveles intermedios: ni existe una despreocupación por las cuestiones metodológicas, ni podemos afirmar que nos encontremos ante el nivel deseable de 
las estrategias metodológicas centradas en la investigación escolar con participación negociada (Hernández de la Torre y Medina, 2014). De este modo, conviven en el aula el diseño de secuencias cerradas y respuestas prescritas, con el desarrollo de actividades improvisadas basadas en el descubrimiento de la realidad.

Tutiaux-Guillón (2003), recoge los resultados de varias investigaciones donde se concluye que la intención de los docentes de Ciencias Sociales de formar ciudadanos democráticos en el aula choca en muchas ocasiones con una práctica que no favorece dicho modelo de ciudadanía. Sin embargo, en nuestro caso, durante el desarrollo de las clases, el ejercicio democrático y crítico está muy presente, por lo que lo situamos en un nivel intermedio de desarrollo profesional en relación con la hipótesis de progresión que utilizamos como referencia.

\section{Referencias}

Arthur, J., Davies, I., \& Hahn, C. (2008) (Eds.). The SAGE Handbook of Education for Citizenship and Democracy. London: SAGE.

Britz, J. (2004). Histoire et mémoire-éducation à la prévention des crimes contre l'humanité. Bruselas: Conseil de l'Europe.

Cuesta, R. (2011). Historia con memoria y didáctica crítica. Con-ciencia Social, 15, 15-30.

De Alba, N., García Pérez, F. F., \& Santisteban, A. (2012). Educar para la ciudadanía en la enseñanza de las Ciencias Sociales. En N. De Alba, F. F. García Pérez, \& A. Santisteban [Eds.] FALTA EL NOMBRE DEL LIBRO (pp. 521-526). Sevilla: Díada Editora.

Delgado Algarra, E. J. (2014). Educación para la ciudadanía en la enseñanza de las Ciencias Sociales y su vinculación con las dimensiones de la memoria: Estudio de caso en ESO (Tesis Doctoral). Recuperado de http://hdl.handle.net/10272/8841

De Miguel, M. (2015). Ideología y pedagogía empírica: Cuestiones para un debate. Revista de Investigación Educativa, 33(2), 269-287.

Díaz Rubiano, M., Fernández Armijo, M. I., Jiménez Maqueda, M. A., Del Pino, F. J., \& Vidal, F. (2008). Unidad 9: España: primer tercio del siglo XX y unidad 14: España: Dictadura y Democracia En $4^{\circ}$ Secundaria. Historia. Edición Andalucía. Oxford Education.

Estepa, J. (2009). La educación del patrimonio y la ciudadanía europea en el contexto español. En R. M. Ávila, B. Borghi, \& I. Mattozzi (Coords.), La educación de la ciudadanía europea y la formación del profesorado. Un proyecto educativo para la "Estrategia de Lisboa". Atti XX Simposio Internacional de Didáctica de las Ciencias Sociales: I Convegno Internazionale Italo-Spagnolo di Didattica delle ScienzeSociali, Bologna, 31 marzo-3 aprile 2009.

Estepa, J. (Ed.) (2013). La educación patrimonial en la escuela y el museo: investigación y experiencias. Huelva: Universidad de Huelva.

Evans, E. (2010). Orientaciones metodológicas para la investigación-acción. Perú: Ministerio de Educación.

Glaser, B., \& Strauss, A. L. (1967). The Discovery of Grounded Theory: Strategies for Qualitative Research. Chicago: Aldine De Gruyter.

Hernández de la Torre, E. \& Medina, P. (2014). Análisis de los obstáculos y barreras para el cambio y la innovación en colaboración en los centros de secundaria: estudio de caso. Revista de Investigación Educativa, 32(2), 269-287. 
Ienaga, S. (2001). Japan's Past, Japan's Future: One Historian's Odyssey (Asian Voices). United States of America: Rowman \& Littlefield.

Kelly, T. (1986). Discussing controversial issues: Four perspectives on the teacher's role. Theory and Research in Social Education, 14, 499-512.

Lässig, S. (noviembre, 2009). History School Textbooks as a Means of Reconciliation?. Some Remarks on Bilateral Textbooks and "Common Histories". Trabajo presentado en International Seminar History and Social Science Textbook, Santiago de Chile.

López Facal, R. (2011). Aprender de los conflictos. Íber, 69, 5-7.

Mainer, J. (julio, 2010). Memoria, historia y didáctica crítica. Los deberes de la educación histórica; por una historia con memoria. Trabajo presentado en XV Congreso Colombiano de Historia, Bogotá.

Mattozzi, I. (2008). Memoria y formación histórica. La memoria en la clase de Historia. Íber, 55, 30-42.

McCrary, N. (2002). Investigating the Use of Narrative in Affective Learning on issues of Social Justice. Theory and Research in Social Education, 30(2), 255-273.

Mejía, W. (noviembre, 2009). What Criticisms are made to the Content of History Science Textbooks?. Trabajo presentado en International Seminar History and Social Science Textbook, Santiago de Chile.

Mouffe, C. (1999). El retorno de lo político: Comunidad, ciudadanía, pluralismo, democracia radical. Barcelona: Paidós.

Pagés, J. (2008). El lugar de la memoria en la enseñanza de la Historia. Íber, 55, 43-53.

Pagés, J., \& Oller, M. (2007). Las representaciones sociales del derecho, la justicia y la ley de un grupo de adolescentes catalanes de $4^{\circ}$ de ESO. Enseñanza de las Ciencias Sociales, 6, 3-19.

Patton, M. Q. (1987). How to Use Qualitative Methods in Evaluation. California: Sage Publications, Inc.

Patton, M. Q. (2002). Qualitative research and evaluation methods. Thousand Oaks, CA: Sage.

Rinaldi, G. (2008). Storia e memoria. En L. Ziruolo (Ed.). I luoghi, la storia, la memoria. Genova: Le Mani.

Santisteban, A. (2007). Una investigación sobre cómo se aprende a enseñar el tiempo histórico. Enseñanza de las Ciencias Sociales, 6, 19-29.

Schugurensky, D. (2009). Citizenship Participation and its Discontents. En K. Daly, D. Schugurensky, \& K. Lopes (Eds.), Learning Democracy by doing. Alternative Practices in Citizenship Learning and Participatory Democracy. Toronto: University of Toronto.

Schugurensky, D., \& Myers, P. (2003). Learning to Teach Citizenship: A Lifelong Learning Approach. Encounters on Education, 4, 145-166.

Schulz, W., Fraillon, J., Ainley, J., Bruno, L., \& Kerr, D. (2008). International Civic and Citizenship Education Study: Assessment Framework. Amsterdam: International Association for the Evaluation of Educational Achievement.

Fecha de recepción: 9 de abril de 2015.

Fecha de revisión: 9 de abril de 2015.

Fecha de aceptación: 29 de marzo de 2016. 